\title{
Aspectos relevantes de la preparación para carillas anteriores de porcelana: Una revisión
}

\author{
Relevant aspects of tooth preparation for anterior porcelain veneers. A review. \\ Gabriela Isabel Ortiz-Calderón ${ }^{1, a, b}$, Luis Gómez-Stella ${ }^{1, a, c, d}$
}

\section{RESUMEN}

Debido a la gran acogida de las carillas de porcelana para la rehabilitación estética y funcional del paciente en el sector antero superior, es importante conocer qué tipo de preparación realizar ya que la terminación palatina influye en la supervivencia de estas restauraciones, así como de la estructura remante dentaria. La literatura describe tres tipos de preparación para las carillas anteriores, tipo "ventana" o intra esmalte, la cual se limita sólo al desgaste del esmalte de la pieza de 0,3 a $0,5 \mathrm{~mm}$, la preparación tipo "pluma" o con reducción incisal, la cual tiene un mini chamfer incisal de hasta $1 \mathrm{~mm}$, y la preparación tipo "overlap" o con solapa incisal que, además del desgaste vestibular y reducción incisal, tiene una preparación de tipo chamfer palatino, llegando así a una preparación incisal total de $2 \mathrm{~mm}$. De acuerdo a los artículos revisados, se sugiere que la preparación más favorable que indica más tasa de supervivencia a largo plazo es la tipo "overlap" o solapa incisal, debido a que favorece a la retención de la carilla y a la distribución homogénea de las fuerzas oclusales. Es importante considerar que mantener la integridad de la estructura dental remanente en boca es la finalidad de todo tratamiento protésico, es por este motivo que las carillas anteriores de porcelana son consideradas como una alternativa de tratamiento conservadora no sólo con fines estéticos, sino también para reestablecer la función.

\section{PALABRAS CLAVE: Coronas con frente estético, preparación prostodóncica del diente}

\section{SUMMARY}

Due to the great success of porcelain veneers for the esthetic and functional rehabilitation of the patient in the upper anterior sector, it is important to know which type of preparation should be done because the palatal margin influences in the survival of these restorations and of the remaining tooth structure. The literature describes three types of preparation for anterior veneers, "window" type or intra enamel, in which the enamel preparation is limited only from 0.3 to $0.5 \mathrm{~mm}$, the "feather" type or with incisal reduction, which has a mini incisal chamfer up to $1 \mathrm{~mm}$, and the "overlap" type or incisal overlap that, in addition of the enamel and incisal reduce, it has a palatal chamfer, reaching a total incisal preparation of $2 \mathrm{~mm}$. According to the reviewed articles, it is suggested

\footnotetext{
Cirujano Dentista.

Residente del Programa de Posgrado en Rehabilitación Oral.

Especialista en Rehabilitación Oral.

Docente auxiliar en Posgrado.
} 
that the most favorable preparation that allows a further rate of long-term survival is the "overlap" or incisal overlap type, because it facilitates the retention of the veneer and the distribution of oclusal forces. It is important to consider that the main purpose of every prosthetic treatment is the maintenance of the integrity of the remaining tooth structure in the mouth. For this reason, the anterior veneers are considered as an alternative to conservative treatment not only for aesthetic purposes, but also to restore the function.

\section{KEYWORDS: Dental veneers, tooth preparation.}

\section{INTRODUCCIÓN}

La idea que surgió del Dr. Charles Pincus en el año 1938, conocido por estar relacionado con la industria cinematográfica de aquella época, era brindar a los artistas una sonrisa bella y estética durante las filmaciones de sus películas, por ello desarrolló unas láminas delgadas de acrílico las cuales eran fijadas temporalmente en los dientes anteriores sin preparaciones por medio de polvos adhesivos para prótesis total, y luego estas tenían que ser retiradas luego de cada filmación ya que no existía ningún sistema adhesivo en esa época que las retenga permanentemente (1-4).

Aunque el resultado estético era muy bueno, esta técnica tenía muchas limitaciones e impedimentos, sobretodo hablando del tema de la adhesión, por lo tanto, poco a poco fue cayendo en desuso (3).

Sin embargo, gracias al avance y el desarrollo de materiales cerámicos para la confección de estos laminados, así como de la técnica del grabado ácido, desarrollada por Buonocuore en 1955 y la introducción de las resinas Bis-GMA por Bowen en 1963, se inició una nueva era en la Odontología $(2,3)$.

En 1975, Rochette fue el primero en proponer el uso de restauraciones de porcelana adheridas a los dientes anteriores. Su técnica fue el grabado ácido de la restauración y la colocación del adhesivo de la superficie dental, usando como cemento una resina, esta fue la base para el desarrollo de las técnicas adhesivas actuales de las restauraciones de porcelana, y es así como nacen las Carillas laminadas de porcelana $(5,6)$.

Más tarde, Horn, así como Calamia y Simonsen , citados por Kina y Bruguera y Peumans y col., documentaron técnicas de tratamiento y adhesión de superficies cerámicas, las cuales finalmente demostraron que sí es posible conseguir una buena adhesión de las carillas de porcelana a la estructura dentaria por medio del grabado con ácido fluorhídrico, el silanizado de la carilla de porcelana y el uso de un cemento resinoso $(3,4)$.

Con el uso de estos procedimientos se puede conseguir alta estética y rehabilitar la función en el sector antero superior.

\section{Carillas de Porcelana - Definición}

Las carillas de porcelana, o también conocidas como laminados cerámicos, son estructuras cerámicas con excelentes características químicas y físicas, asociadas adhesivamente a las estructuras dentales, brindando así protección mecánica recíproca entre el diente y la restauración (3).

El objetivo que se le atribuye a una carilla de porcelana desde el punto de vista estético es lograr una armonía en la sonrisa y de esa manera conseguir que el paciente logre la confianza en sí mismo y el desarrollo de su personalidad (6); además de la recuperación de la función, gracias al reestablecimiento de la guía anterior y de la guía canina (8) brindando el largo adecuado de los dientes anteriores, como lo reportó Friedman $(7,10)$.

Las carillas son una alternativa restauradora conservadora ya que evita el desgaste excesivo del diente a comparación de la reducción que se hace para una corona convencional, manteniendo así buena parte de la estructura dentaria, sólo retirándose entre 3\% y 30\% de la estructura total del diente $(3,6,9)$.

El éxito de las carillas de porcelana ha sido reportado a un $93 \%$ después de 15 años (11), y se determina por la resistencia y la durabilidad de la adhesión lograda por la superficie dentaria tratada, el cemento resinoso y la carilla de porcelana igualmente tratada $(3,4)$, así como otros factores que ayudan a mejorar su com- 
portamiento mecánico, como el diseño apropiado de la preparación y la conservación de la estructura dentaria remanente $(10,11)$; tiene como ventajas su alta apariencia estética, su probada biocompatibilidad, y su predictibilidad a largo plazo (8); sin embargo, las fallas más comunes asociadas a las carillas son la fractura y la descementación, siendo el margen gingival y el área incisal las zonas mayormente afectadas (10).

\section{Indicaciones y Contraindicaciones}

Las carillas de porcelana ofrecen soluciones conservadoras y estéticamente aceptables para situaciones clínicas como los oscurecimientos moderados, hipoplasia del esmalte y malformaciones, cierre de diastemas, malposiciones dentarias leves que no requieran un tratamiento de Ortodoncia, cambio de restauraciones antiguas anteriores antiestéticas, desgaste dentario fisiológico por la edad, devolución de la guía anterior, entre otras $(6,7)$. Están contraindicadas en casos de insuficiente cantidad de esmalte, hábitos orales que no se puedan controlar y una oclusión desfavorable como mordidas vis a vis y mordidas cruzadas debido al estrés excesivo durante la función $(6,7)$.

\section{Preparación para Carillas}

Inicialmente, estas carillas eran colocadas en los dientes sin preparación alguna (9). Los avances de las técnicas adhesivas han ayudado a mejorar la fuerza adhesiva entre la carilla y el diente, pero se observó que las fracturas en la cerámica ocurrían generalmente en el borde incisal debido al gran estrés en esa zona (7). Por ello, a pesar de que no está estipulado un consenso en cuanto a preparar el diente o no para recibir una carilla, se ha recomendado las preparaciones más conservadoras (9), incluso algunos autores sugieren no realizar preparación de la superficie dentaria (4). Sin embargo, siempre se consideró que un chamfer palatino era necesario para brindar mayor resistencia a las carillas (7), e incluso estudios actuales afirman que la reducción de esmalte es necesaria para mejorar la adhesión, pero tomando en cuenta que esta preparación no debe de ir más allá de la superficie del esmalte (4).

La mayoría de dientes que van a recibir una carilla de porcelana deben de tener una remoción de esmalte de la cara vestibular, usualmente de $0,5 \mathrm{~mm}$ aproximadamente, lo cual permite y brinda el grosor mínimo de la futura carilla (4). Christensen, citado por Peumans y col en el año 2000 (4), afirma que la cantidad óptima de remoción de esmalte que debe hacerse es de $0.75 \mathrm{~mm}$, sin embargo, de acuerdo a Ferrari y col., citados también por Peumans y col., en su revisión del año 2000 (4), esa reducción en la parte gingival de la cara vestibular de los dientes anteriores no se puede alcanzar sin haber llegado ya a dentina (4). La preparación dentaria está orientada a controlar el sobrecontorneo, distribuir el estrés y facilitar la ejecución de la técnica (10).

McLaughlin y Morrison, citados por Nattress y col., en 1995, mencionan las características básicas a tener en cuenta para realizar este tratamiento, así: la preparación debe ser conservadora permitiendo un recubrimiento aproximado de $0,5 \mathrm{~mm}$ de porcelana sin dar al diente una apariencia voluminosa logrando un margen gingival higiénico, evitar abarcar la dentina, especialmente en los bordes de la preparación, y no debe incluir ángulos internos para así permitir un correcto asentamiento sin ninguna interferencia (12). Según la literatura, existe tres tipos básicos de preparación incisal para carillas (Figura 1) $(4,13)$ :

Preparación de tipo "ventana" o preparación intra-esmalte (Figura 1(a))

Esta preparación está indicada en casos con alteración leve del color, el desgaste que se hace en la cara vestibular y proximal es de $0,3 \mathrm{~mm}$ a $0,5 \mathrm{~mm}$, lo más uniforme posible. No se realiza reducción del borde incisal. Su ventaja es la buena mimetización con la estructura dentaria y evita el sobrecontorneo de la restauración. Estudios in vitro de análisis del elemento finito demuestran que este tipo de preparación se comporta al igual que un diente anterior antes las cargas $(13,14)$.

Preparación de tipo "pluma" o con reducción incisal (Figura 1(b))

El desgaste vestibular también es de $0.3 \mathrm{~mm}$ a 0.5 $\mathrm{mm}$. El desgaste incisal es de $1 \mathrm{~mm}$, a manera de un pequeño chamfer. Es un diseño funcional ya que está indicado cuando se necesita alargar la zona incisal del diente, sin embargo, este alargamiento no debe de ser mayor a $2 \mathrm{~mm}$ porque se crearía un efecto de palanca. Según algunos estudios in vitro, la mayor carga de estrés se puede concentrar en la interfase restauración - diente, es por esto que es necesario verificar la oclusión antes de la preparación $(7,9,13-15)$.

Preparación de tipo "overlap" o solapa incisal (Figura 1(c)) 


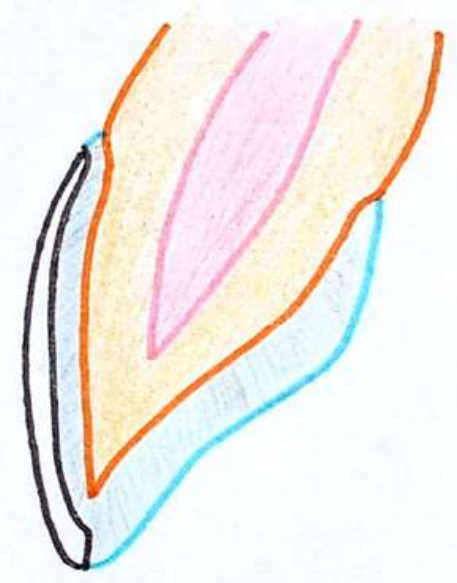

No reducción del borde incisal

Figura.1a. Tipo "ventana".

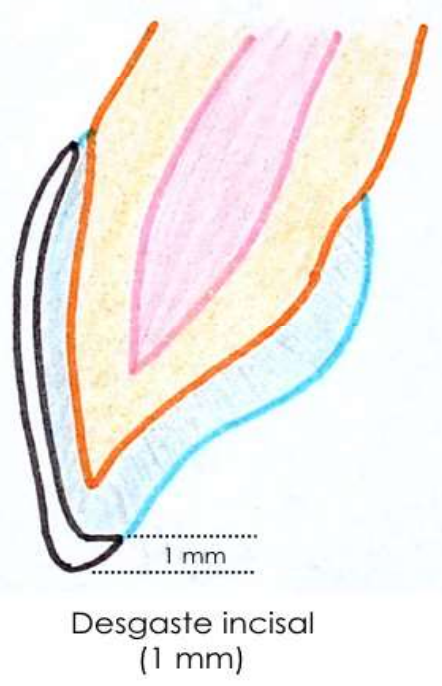

Figura.1b. Tipo "pluma”.

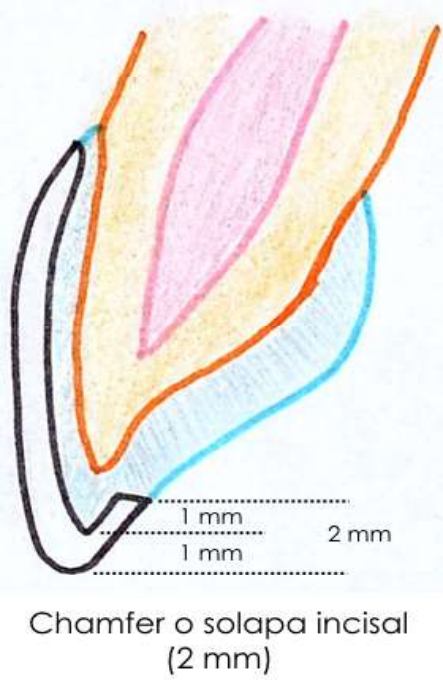

Fig.1c. Tipo "overlap"

Figura 1. Terminaciones palatinas en la preparación para carillas de porcelana.

Modificación de: Baratieri L, et.al. Odontologia Restauradora Fundamentos e Possibilidades. 1ra ed.

São Paulo: Santos; 2001. (13)

Igualmente la reducción vestibular se encuentra en el rango de $0.3 \mathrm{~mm}$ a $0.5 \mathrm{~mm}$. La reducción incisal es de $1 \mathrm{~mm}$ en longitud, y $1 \mathrm{~mm}$ hacia la cara palatina, creando un chamfer palatino o solapa incisal de $2 \mathrm{~mm}$. Este diseño es el mejor según la mayoría de estudios, ya que muestra mayor resistencia a la fractura gracias a la solapa incisal, que le da más soporte dentario y mejor distribución de cargas y por ello está indicado para devolver la guía anterior $(7,9,11,13,14,16)$.

Actualmente, no existe un consenso en la literatura sobre una preparación apropiada para carillas. Las distintas situaciones clínicas que presenten los pacientes, incluso si ya estos dientes presentan desgaste influye en la decisión de la reducción o no reducción del borde incisal, siendo ésta una de las posibles variables que afectan el éxito de las restauraciones de carillas de porcelana $(11,17)$.

Estudios in vitro evalúan el estrés dinámico y el estrés fotoelástico de dos dimensiones demostrando que la preparación de tipo "overlap" es más favorable debido a la distribución de la fuerza oclusal sobre una superficie más amplia, reduciendo así la concentración de estrés entre la carilla y el diente $(10,17)$.

\section{DISCUSIÓN}

Li y col., demostraron en un estudio de elemento finito simulando la inclinación de las piezas y las fuerzas oclusales, que la preparación de "overlap", o también llamada chamfer palatino, era favorable para las piezas restauradas con carillas de porcelana ya que distribuían y toleraban mejor el estrés mecánico bajo las cargas funcionales (10), esto indica la preferencia por esta preparación, ya que mejora la resistencia mecánica de la carilla, lo cual observamos en varios estudios de elemento finito; incrementando la superficie de adhesión y retención de la restauración $(16,17)$.

Por el contrario, Hui y col., demostraron in vitro que las preparaciones para carillas sin reducción incisal eran más resistentes que las preparaciones con solapa o sobre pase incisal (17), al igual que los resultados obtenidos por Granell-Ruiz y col., en el 2010, que evaluaron 323 carillas cementadas en 70 pacientes entre los años 1995 y 2003, de las cuales, 124 fueron del tipo de preparación "ventana", que comprende sólo la cara vestibular del diente, y 199 fueron del tipo "overlap" o solapa incisal, con un chamfer palatino de $1 \mathrm{~mm}$. Luego de las evaluaciones y contro- 
les se encontraron un total de 13 fracturas (4\%), 11 $(84,6 \%)$ ocurrieron en los diseños funcionales, y 2 $(15,4 \%)$ en los diseños simples (18). Los hallazgos de Beier y col., en el 2012 fueron similares. Los autores realizaron un estudio clínico retrospectivo para evaluar el comportamiento de las carillas de porcelana en 292 dientes vitales, con preparaciones tipo "overlap" o solapa incisal y ventana (sin sobrepase incisal) en 74 pacientes, cementadas entre noviembre de 1987 y diciembre del 2009. Realizaron ajustes de la oclusión luego de la colocación de las carillas y controles periódicos. Los resultados mostraron que a medida de que pasaban los años, disminuía la tasa de éxito en los dientes con preparación "overlap", mientras que en los dientes con preparación de tipo "ventana" se mantenía siempre al $100 \%$ (17), estos resultados pueden deberse a que estos estudios son in vivo, y como se sabe, las fuerzas reales de la masticación son mayores, siendo este un factor importante a tener en cuenta sobretodo en pacientes con mayores fuerzas oclusales, en los cuales se podría optar por preparaciones de tipo "ventana".

$\mathrm{Al}$ analizar los resultados presentados, se sugiere que la terminación incisal no es el factor determinante del éxito o fracaso de las carillas, también influyen otras variables como la vitalidad de los dientes, la fuerza de adhesión, el uso de porcelanas de alta resistencia o de porcelanas feldespáticas convencionales, la necesidad de rehabilitar la guía anterior, el lograr una adecuada adhesión y la dirección de las fuerzas oclusales. En este último punto es importante considerar las fuerzas de cizallamiento en los dientes anteriores y el punto de acople con sus antagonistas, que no debe interferir en la interfaz carilla-diente además de la historia de bruxismo del paciente $(17,18)$.

Varios autores refieren que la adhesión es óptima en esmalte, y es por esto que el rango de preparación que se recomienda va desde no realizar preparación hasta los $0.75 \mathrm{~mm}$ en vestibular. Este también es un factor que influye de forma importante en la tasa de éxito de las carillas, independientemente de la reducción o no reducción incisal, ya que estando cementadas en esmalte se aumenta la resistencia a la adhesión del cemento resinoso a la superficie de esmalte grabada luego de la preparación, se evita el sobre contorneo al dar el espacio necesario para la restauración y se controla la distribución de estrés en la carilla (12).

En un estudio de Nattress y col., se observó que usualmente al hacer las preparaciones para carillas de porcelana sin guías, se tiende a desgastar más unas zonas (proximal y cervical hasta $1,2 \mathrm{~mm}$ ) exponiendo así mayor dentina, y en otras zonas no se consigue el desgaste ideal (incisal medio hasta 0,12 mm). Es decir, no se consigue un desgaste uniforme ni el espacio adecuado necesario, por lo que se recomienda usar matrices de silicona basadas en el encerado para el diagnóstico o alternarlo con surcos guías para facilitar y ayudar en la preparación dentaria, tanto en la cara vestibular como en el borde incisal, de ser necesario (12).

Las preparaciones de tipo "ventana" son las ideales porque sólo abarcan el esmalte dentario, lo cual es muy favorable para la adhesión, también se han reportado buenos valores de resistencia a la fractura, pero hay que considerar que la restauración que se obtendrá será muy delgada al igual que la fina capa de cemento que se tendrá, incrementando el estrés de contracción y al ser sometida a las cargas oclusales podría tener mayor riesgo de fractura (11); esto brinda una razón más por la cual varios autores sugieren que la extensión palatina es más recomendada ya que distribuye mejor las cargas oclusales $(11,10,16,17)$.

Schmidt y col., realizaron un estudio in vitro en el que evaluaron la resistencia a la fractura de las carillas de porcelana cementadas en piezas anteriores con preparaciones de tipo "overlap" (chamfer palatino) y de tipo "pluma" (sólo reducción incisal), sometidas a cargas, y los resultados que se obtuvieron fueron que las piezas con chamfer palatino resistieron las mayores cargas $(166,67 \mathrm{~N})$ a comparación de las piezas con sólo reducción incisal $(131,84 \mathrm{~N})$, incluso en piezas que ya presentan un desgaste, recomiendan las preparaciones con chamfer palatino para incrementar la resistencia a la fractura, basándose en que las fuerzas oclusales que se encuentran en los dientes anteriores son de aproximadamente $130 \mathrm{~N}$ (11).

Los beneficios que se obtienen al utilizar un chamfer palatino son que la cerámica extendida hacia palatino actúa como una llave, reteniendo la restauración al diente ya que se aumenta la superficie adhesiva de la pieza y además se distribuye mejor toda la fuerza oclusal sobre toda esta superficie (11).

Se debe de tener en cuenta que si se trata de alargar la corona dentaria, se recomienda hacer el desgaste incisal y optar por la preparación de tipo "overlap", para 
así conseguir una mayor supervivencia de este tipo de preparación de restauraciones conocidas también como "funcionales" $(17,18)$, sin embargo, ya que la tasa de supervivencia de los diseños simples es mayor (94\%) que en los diseños funcionales (85\%) al igual que lo que se encuentra en otros estudios in vitro (19), Fradeani y col., citados por Stappert y col., y Magne y col., sugieren optar por el diseño de las restauraciones funcionales de reducción incisal sin el chamfer palatino $(14,15)$.

De acuerdo al estudio de elemento finito de Magne y Douglas, lo que lleva a determinar si es necesario realizar la reducción incisal y un chamfer ("overlap") o mini chamfer ("pluma") es la cantidad de estructura remanente de diente natural (14):

Si se tiene suficiente superficie, debe evitar extenderse demasiado hacia palatino ya que se concentrará mayor estrés en esa zona innecesariamente, preferir un mini chamfer o una preparación tipo "ventana", según lo que requiera el caso.

Si la cantidad de remanente dentario es moderada, es decir, se tiene el tercio cervical y medio de la corona dentaria, no se recomienda hacer la preparación de tipo "overlap" ya que esa zona aún es de alto estrés, en estas situaciones lo que se puede hacer es limitar la extensión de la cerámica hasta el margen palatino, para así evitar cargar la interfase.

Si existe desgaste severo o fractura amplia que abarcan el tercio cervical o tal vez hasta la mitad del tercio medio, se puede emplear un mini chamfer ya que en esta zona no se concentra la gran cantidad de estrés, a diferencia del tercio incisal y medio.

\section{CONCLUSIONES}

Considerando que hay estudios in vitro que afirman que no hay diferencias significativas entre los distintos tipos de preparaciones dentarias para las carillas de porcelana $(15,20)$, se llega a la conclusión que si lo que se necesita es mejorar la estética, la preparación de tipo "ventana" cumple con ese requerimiento, resistiendo cargas al semejarse al comportamiento natural del diente, evitando así mayor desgaste dentario $(11,16)$. Hay también estudios retrospectivos que evalúan la resistencia y longevidad de estas restauraciones, mencionando que si hay diferencias, tal vez no tan significativas en algunos casos $(21,22,23)$, muchos de estos afirman y sugieren que la preparación de tipo "overlap" o solapa incisal cuando se necesite recuperar la funcionalidad del diente, ya que es la que va a dar mejores resultados y mayor rango de supervivencia gracias a todas las características antes mencionadas $(7,9,11,14,16)$.

\section{Correspondencia:}

Gabriela Isabel Ortiz Calderón

Correo electrónico: gabriela_oc7@hotmail.com

\section{REFERENCIAS BIBLIOGRÁFICAS}

1. Goldstein RE. Esthetics in dentistry. 2nd ed. USA: B.C. Decker; 1998.

2. Henostroza G, Dell'Acqua A, Espinosa R, et al. Estética en odontología restauradora. 1era ed. Madrid: Ripano S.A; 2006.

3. Kina S, Bruguera A. Invisible: Restauraciones estéticas cerámicas. 1 ed. São Paulo: Artes Médicas; 2008.

4. Peumans M, Van Meerbeek B, Lambrechts P, Vanherle G. Porcelain Veneers: A review of the literature. J Dent. 2000; 28(3): 163-177.

5. Magne P, Belser U. Restauraciones de porcelana adherida en los dientes anteriores: Método Biomimético. Barcelona: Quintessence; 2004.

6. Mathew C, Sebeena M, Karthik K. A review on ceramic laminate veneers. J Indian Acad Dent Spec Res. 2010; 1(4): 33-37.

7. Jankar A, Kale Y, Kangane S, Ambekar A, Sinha M, Chaware S. Comparative evaluation of fracture resistance of Ceramic Veneer with three different incisal design preparations - An in-vitro study. J Int Oral Health. 2014; 6(1): 48-54.

8. Mezzomo E, Chiapinotto G, Da Fontoura L, Oppermann R, Graça M, Puricelli E. Rehabilitación Oral para el Clínico. 1 ed. São Paulo: Actualidades Médico Odontológicas Latinoamérica; 1997.

9. Shetty A, Kaiwar A, Shubhashini N, et al. Survival rates of porcelain laminate restoration based on different incisal preparation designs: An analysis. J Conserv Dent. 2011; 14(1): 10-15.

10. Li Z, Yang Z, Zuo L, Meng Y. A three-dimensional finite element study on anterior laminate veneers with different incisal preparations. J Prosthet Dent. 2014; 112(2): 325-333.

11. Schmidt K, Chiayabutr Y, Phillips K, Kois J. Influence of preparation design and existing condition of tooth 
structure on load to failure of ceramic laminate veneers. J Prosthet Dent. 2011; 105(6): 374-382.

12. Nattress B, Youngson C, Patterson C, Martin D, Ralph $\mathrm{J}$. An in vitro assessment of tooth preparation for porcelain veneer restorations. J Dent. 1995; 23(3): 165170 .

13. Baratieri L, Nunes C, Alves C, et al. Odontologia restauradora fundamentos e possibilidades. 1 ed. São Paulo: Santos; 2001.

14. Magne P, Douglas W. Design optimization and evolution of bonded ceramics for the anterior dentition: a finite-element analysis. Quintessence Int. 1999; 30(10): 661-672.

15. Stappert C, Ozden U, Gerds T, Strub J. Longevity and failure load of ceramic veneers with different preparation designs after exposure to masticatory simulation. J Prosthet Dent. 2005; 94(2): 132-139.

16. Cötert H, Dündar M, Ostürk B. The effect of various preparation designs on the survival of porcelain laminate veneers. J Adhes Dent. 2009; 11(5): 405-411.

17. Beier US, Dhima M, Koka S, Salinas T, Dumfahrt H. Comparison of two different veneer preparation designs in vital teeth. Quintessence Int. 2012; 43(10): 835-839.

18. Granell M, Fons A, Labaig C, Martínez A, Román J, Solá M. A clinical longitudinal study 323 porcelain laminate veneers. Period of study from 3 to 11 years. Med Oral Patol Oral Cir Bucal. 2010; 15(3): 531-537.

19. Hahn P, Gustav M, Hellwig E. An in vitro assessment of the strength of porcelain veneers dependent on tooth preparation. J Oral Rehabil. 2000; 27(12): 10241029.

20. Alghazzawi T, Lemons J, Liu P, Essig M, Janowski G. The failure load of CAD/CAM generated zirconia and glass-ceramic laminate veneers with different preparation designs. J Prosthet Dent. 2012; 108(6): 386-393.

21. Zarone F, Apicella D, Sorrentino R, Ferro V, Aversa $\mathrm{R}$, Apicella A. Influence of tooth preparation design on the stress distribution in maxillary central incisors restored by means of alumina porcelain veneers: a 3D-finite element analysis. Dent Mater. 2005; 21(12): 1178-1188.

22. Meijering A, Creugers N, Roeters F, Mulder J. Survival of three types of veneer restorations in a clinical trial: a 2.5-year interim evaluation. J Dent. 1998; 26(7): 563-568.

23. Smales R, Etemadi S. Long-term survival of porcelain laminate veneers using two preparation designs: a retrospective study. Int J Prosthodont. 2004; 17(3): 323-326.

Recibido : 09/03/2015

Aceptado: 27/04/2016 